\title{
Distributed Interference Pricing with MISO Channels
}

\author{
Changxin Shi, Randall A. Berry, and Michael L. Honig \\ Department of Electrical Engineering and Computer Science \\ Northwestern University, Evanston, Illinois 60208 \\ Email: cshi@u.northwestern.edu, \{rberry,mh\}@ece.northwestern.edu
}

\begin{abstract}
We study a distributed algorithm for adapting transmit beamforming vectors in a multi-antenna peer-to-peer wireless network. The algorithm attempts to maximize a sum of per-user utility functions, where each user's utility is a function of his transmission rate, or equivalently the received signalto-interference plus noise ratio (SINR). This is accomplished by exchanging interference prices, each of which represents the marginal cost of interference to a particular user. Given the interference prices, users update their beamforming vectors to maximize their utility minus the cost of interference. For a two-user system, we show that this algorithm converges for a suitable class of utility functions. Convergence of the algorithm with more than two users is illustrated numerically.
\end{abstract}

\section{INTRODUCTION}

Mitigating interference is critical for efficiently sharing wireless spectrum. When nodes are equipped with multiple antennas, the additional spatial degrees of freedom can be exploited to reduce interference. This paper considers such a setting, namely an interference channel consisting of multiinput, single-output (MISO) wireless links. The objective is to select the beamforming vector and transmission power level at each transmitter in an attempt to maximize the overall network performance. Performance is measured in terms of the sum of per user utilities, where each utility is a function of the transmission rate, or equivalently the signal-to-interference plus noise ratio (SINR). In a network without centralized control (e.g., an ad hoc network), maximizing the total utility is complicated due to the interference among users, which causes each user's beamforming vector to affect not only that user's utility but the utility of every other user. Solving this in a scalable manner requires a distributed algorithm with limited information exchange. Such an algorithm is the focus of this paper.

The algorithm we study is motivated by the work in [1] and [2], which consider algorithms for power allocation in single-antenna wireless networks. These asynchronous distributed pricing (ADP) algorithms are based on exchanging interference prices among users ${ }^{1}$, where an interference price is a user's marginal change in utility per unit interference power. Given the interference prices from the neighboring users, each user then optimizes his own utility minus the

This work was supported by the Army Research Office under grant W911NF-06-1-0339 and by NSF under grant CNS-0626558.

${ }^{1}$ We refer to each transmitter-receiver pair as a "user". interference cost to other users. By iteratively updating interference prices and powers, the algorithms in [1], [2] are shown to converge under suitable assumptions on the users' utility functions.

The ADP algorithm for a single-antenna network in [1] can be directly generalized to a MISO network, as shown in Section II. Such an algorithm was previously presented in [3] for the special case of rate utilities (i.e. a user's utility is equal to his transmission rate given by the Shannon capacity formula). Simulation results shown there with two users indicate that this algorithm converges to an allocation that is essentially optimal. However, there is no proof that the algorithm must converge, and it is not clear how it performs with other utility functions. Moreover, the convergence proof in [1] for a single antenna network, which is based on relating this algorithm to best response updates in a supermodular game, does not directly generalize to this setting. Likewise, the convergence proof for the modified algorithm in [2] does not apply here. For a two user MISO interference channel we prove that this algorithm does converge under a similar condition as in [1]. Our initial proof is based on first showing several properties of the optimal beamforming vectors, which enable us to re-parameterize the original optimization problem. The steps in the ADP algorithm can also be written in terms of these re-parameterized values. After doing this, we show that the algorithm again corresponds to best response updates in a supermodular game, under suitable choices of utility functions. We also give an alternative proof that is based on defining a different game in which the strategy of each player is the "interference power" they create at the other user's receiver. With this formulation the algorithm also corresponds to best response updates in a supermodular game.

In terms of related work, in addition to [1], [2], the algorithms in [4] and [5] also exchange information similar to interference prices to facilitate distributed power control in single antenna interference channels. For MIMO interference channels, the most common approaches that have been studied for distributed optimization are based on iterative waterfilling (e.g. [6]-[8]). In these approaches each user iteratively selects their transmit covariance matrix to maximize their rate given the current interference. Such approaches may not converge in general or may converge to a rate-pair that is not Pareto optimal. In [9], it is shown that for a two- 
user MISO interference channel, any Pareto optimal rate-pair can be achieved as a linear combination of a beamformer corresponding to the Nash equilibrium and the zero-forcing beamformer. This is closely related to the decomposition result we give in Section III. However, [9] does not address how to determine this combination in a distributed manner, which is our focus here.

In the next section, we give our system model and generalize the ADP algorithm in [1] to this setting. Section III contains our main analytical results. Simulation results are presented in Section IV, and conclusions are given in Section V.

\section{System Model And Generalized ASYNCHRONOUS DistribUted ALGORITHM}

We consider a MISO wireless network with a narrowband time-invariant wireless channel, in which there are $K$ pairs of transmitters and receivers, each with $N$ transmit antennas sharing a single flat fading channel. The received signal for user $i=1, \ldots, K$ is given by

$$
y_{i}=\mathbf{v}_{\mathbf{i}}^{\dagger} \mathbf{h}_{\mathbf{i i}} x_{i}+\sum_{j \neq i} \mathbf{v}_{\mathbf{j}}^{\dagger} \mathbf{h}_{\mathbf{j i}} x_{j}+n
$$

where $\dagger$ denotes Hermitian transpose, $\mathbf{h}_{\mathbf{i j}}=$ $\left[h_{i j}^{1}, h_{i j}^{2}, \cdots, h_{i j}^{N}\right]^{T}$, is the channel vector from the $i$ th transmitter to the $j$-th receiver through the $N$ antennas, $x_{i}$ is the transmitted symbol of user $i, n$ is additive complex Gaussian noise with variance $n_{0}$, and $\mathbf{v}_{\mathbf{i}}$ is the beamforming vector for user $i$. Assuming the transmit symbol has unit variance for all users, the received SINR for each user $i$ can be written as

$$
\gamma_{i}=\frac{\left|\mathbf{v}_{\mathbf{i}}^{\dagger} \mathbf{h}_{\mathbf{i i}}\right|^{2}}{n_{0}+\sum_{j \neq i}\left|\mathbf{v}_{\mathbf{j}}^{\dagger} \mathbf{h}_{\mathbf{j i}}\right|^{2}} .
$$

The quality of service for each user $i$ is measured via a utility function $u_{i}\left(\gamma_{i}\right)$, which is assumed to be a monotonically increasing and concave function of the received SINR ${ }^{2}$. Our objective is to maximize the total utility over all users' beamforming vectors $\left\{\mathbf{v}_{\mathbf{i}}\right\}$, i.e.,

$$
\begin{aligned}
& \max _{\mathbf{v} \mathbf{1}, \cdots, \mathbf{v}_{\mathbf{K}}} \sum_{i=1}^{K} u_{i}\left(\frac{\left|\mathbf{v}_{\mathbf{i}}^{\dagger} \mathbf{h}_{\mathbf{i i}}\right|^{\mathbf{2}}}{n_{0}+\sum_{j \neq i}\left|\mathbf{v}_{\mathbf{j}}^{\dagger} \mathbf{h}_{\mathbf{j i}}\right|^{2}}\right) \\
& \text { s.t. }\left|\mathbf{v}_{\mathbf{i}}\right|^{2} \leq P_{i}^{\max } \quad \text { for all } i=1, \ldots, K
\end{aligned}
$$

where $P_{i}^{\max }$ denotes a power constraint for user $i$.

Any locally optimal solution $\tilde{\mathbf{v}}_{\mathbf{1}}, \ldots, \tilde{\mathbf{v}}_{\mathbf{K}}$ of Problem $P_{0}$ should satisfy the Karush-Kuhn-Tucker (KKT) conditions [10]. In particular, there must exist unique Lagrange multipliers $\lambda_{i} \geq 0$ associated with each power constraint such

\footnotetext{
${ }^{2}$ Equivalently, one can view each user's utility as a function of his transmission rate given by the Shannon capacity with interference treated as noise.
}

that for all $i=1, \ldots, K$ :

$$
\begin{aligned}
& {\left[\frac{u_{i}^{\prime}}{n_{0}+\sum_{j \neq i}\left|\tilde{\mathbf{v}}_{\mathbf{j}}^{\dagger} \mathbf{h}_{\mathbf{j i}}\right|^{2}}\left(\mathbf{h}_{\mathbf{i i}} \mathbf{h}_{\mathbf{i i}}^{\dagger}\right)\right.} \\
& \left.-\sum_{j \neq i} \frac{u_{j}^{\prime}\left|\tilde{\mathbf{v}}_{\mathbf{j}}^{\dagger} \mathbf{h}_{\mathbf{j}}\right|^{2}}{\left(n_{0}+\sum_{k \neq j}\left|\tilde{\mathbf{v}}_{\mathbf{k}}^{\dagger} \mathbf{h}_{\mathbf{k j}}\right|^{2}\right)^{2}}\left(\mathbf{h}_{\mathbf{i j}} \mathbf{h}_{\mathbf{i j}}^{\dagger}\right)\right] \tilde{\mathbf{v}}_{\mathbf{i}}=\lambda_{i} \tilde{\mathbf{v}}_{\mathbf{i}}
\end{aligned}
$$

where $u_{i}^{\prime}$ denotes the first order derivative of $u_{i}\left(\gamma_{i}\right)$ with respect to $\gamma_{i}$. Note that $u_{i}^{\prime}$ is a function of $\tilde{\mathbf{v}}_{\mathbf{1}}, \ldots, \tilde{\mathbf{v}}_{\mathbf{K}}$ but to simplify notation we do not explicitly denote this.

Following [1], for any given choice of beamforming vectors $\mathbf{v}_{\mathbf{1}}, \ldots, \mathbf{v}_{\mathbf{K}}$, let

$$
\pi_{i}=-\frac{\partial u_{i}}{\partial I_{i}}=\frac{u_{i}^{\prime}\left|\mathbf{v}_{\mathbf{i}}^{\dagger} \mathbf{h}_{\mathbf{i i}}\right|^{2}}{\left(n_{0}+\sum_{j \neq i}\left|\mathbf{v}_{\mathbf{j}}^{\dagger} \mathbf{h}_{\mathbf{j i}}\right|^{2}\right)^{2}}
$$

be the interference price for user $i$, where $I_{i}=\sum_{j \neq i}\left|\mathbf{v}_{\mathbf{j}}^{\dagger} \mathbf{h}_{\mathbf{j i}}\right|^{2}$ is the total interference power for user $i$, which depends on the beamforming vectors of all users other than $i$. Given fixed interference prices and beamforming vectors for every other user, suppose that user $i$ then solves the following subproblem

$$
\begin{gathered}
\max _{\mathbf{v}_{\mathbf{i}}} u_{i}\left(\frac{\left|\mathbf{v}_{\mathbf{i}}^{\dagger} \mathbf{h}_{\mathbf{i i}}\right|^{\mathbf{2}}}{n_{0}+\sum_{j \neq i}\left|\mathbf{v}_{\mathbf{j}}^{\dagger} \mathbf{h}_{\mathbf{j i}}\right|^{2}}\right)-\sum_{j \neq i} \pi_{j}\left|\mathbf{v}_{\mathbf{i}}^{\dagger} \mathbf{h}_{\mathbf{i j}}\right|^{2} \\
\text { s.t. }\left|\mathbf{v}_{\mathbf{i}}\right|^{2} \leq P_{i}^{\text {max }} .
\end{gathered}
$$

It can be seen that if the other users' interference prices and beamforming vectors are set at their optimal values, then the KKT condition of this subproblem matches the $i$-th KKT condition for Problem $P_{0}$. In other words, some locally optimal action for user $i$ in this subproblem will also be the same as that user's action in the globally optimal solution of Problem $P_{0}$.

The previous observations lead to a natural generalization of the ADP algorithm in [1] to this setting. Namely, we allow each user to iteratively update their beamforming vectors by solving Problem $P_{i}$ and then announce new interference prices. Formally, this algorithm is described as follows:

1) Each user $i$ chooses an initial beamforming vector $\mathbf{v}_{\mathbf{i}}$ satisfying the power constraint.

2) Using (4), each user $i$ calculates the interference price $\pi_{\mathbf{i}}$ given the current beamforming vectors and announces this price to every other user.

3) Periodically, each user $i$ solves Problem $P_{i}$ and updates his beamforming vector, given all the other users' interference prices $\left\{\pi_{j}\right\}_{j \neq i}$.

4) Go to step 2 and repeat.

We refer to this as the MISO-ADP algorithm. Steps 2 and 3 of the algorithm may be performed asynchronously among the users. Note that each user only announces a single interference price. In addition to these prices, user $i$ 's calculations in this algorithm only require knowledge of that user's SINR, that user's received signal power $\left|\mathbf{v}_{\mathbf{i}}^{\dagger} \mathbf{h}_{\mathbf{i i}}\right|^{2}$, and the channel gains $\mathbf{h}_{\mathrm{ij}}$ for all $j$. In particular, there is no need to know the other users' beamforming vectors or the channel gains $\mathbf{h}_{\mathbf{k j}}$ for $k \neq i$.

The MISO-ADP algorithm gives a distributed approach for adapting each user's beamforming vector with limited 
information exchange. However, there are two key questions. First, how difficult is it to solve the optimization problem in step 3? Second, does this algorithm converge? From our previous arguments, it follows that if the algorithm converges, then the limit point will satisfy the KKT conditions of Problem $P_{0}$. Furthermore, if there is a unique solution to the KKT conditions, then this point will be globally optimal. Next, we provide answers to these questions for a two-user interference channel.

\section{Two-USER MISO INTERFERENCE CHANNEL}

In this section we focus on the case of $K=2$ users. To begin, we show a key structural property of the solution to Problem $P_{0}$, which enables us to re-parameterize this problem in terms of a single "angle variable" for each user. We then re-cast the MISO-ADP algorithm in terms of this parametrization and answer the two questions raised in the previous section. At last, we provide an alternative proof of the convergence by introducing interference power.

\section{A. Angle Parametrization}

Consider Problem $P_{0}$ with $K=2$ users, so that the optimization variables are $\mathbf{v}_{\mathbf{1}}$ and $\mathbf{v}_{\mathbf{2}}$. The KKT conditions (3) for this case can be re-written as:

$\left[\frac{u_{i}^{\prime}}{n_{0}+\left|\tilde{\mathbf{v}}_{\mathbf{j}}^{\dagger} \mathbf{h}_{\mathbf{j i}}\right|^{2}}\left(\mathbf{h}_{\mathbf{i i}} \mathbf{h}_{\mathbf{i i}}^{\dagger}\right)-\frac{u_{j}^{\prime}\left|\tilde{\mathbf{v}}_{\mathbf{j}}^{\dagger} \mathbf{h}_{\mathbf{j} \mathbf{j}}\right|^{2}}{\left(n_{0}+\left|\tilde{\mathbf{v}}_{\mathbf{i}}^{\dagger} \mathbf{h}_{\mathbf{i j}}\right|^{2}\right)^{2}}\left(\mathbf{h}_{\mathbf{i j}} \mathbf{h}_{\mathbf{i j}}^{\dagger}\right)\right] \tilde{\mathbf{v}}_{\mathbf{i}}=\lambda_{i} \tilde{\mathbf{v}}_{\mathbf{i}}$

for $i=1,2$ and $j \neq i .^{3}$ This is a nonlinear equation in the beamforming vectors. However, given any fixed $\tilde{\mathbf{v}}_{\mathbf{1}}$ and $\tilde{\mathbf{v}}_{\mathbf{2}}$ that satisfy (5), for each $i, \tilde{\mathbf{v}}_{\mathbf{i}}$ must be an eigenvector of the matrix

$$
A_{i}=\left[\frac{u_{i}^{\prime}}{n_{0}+\left|\tilde{\mathbf{v}}_{\mathbf{j}}^{\dagger} \mathbf{h}_{\mathbf{j i}}\right|^{2}}\left(\mathbf{h}_{\mathbf{i i}} \mathbf{h}_{\mathbf{i i}}^{\dagger}\right)-\frac{u_{j}^{\prime}\left|\tilde{\mathbf{v}}_{\mathbf{j}}^{\dagger} \mathbf{h}_{\mathbf{j}}\right|^{2}}{\left(n_{0}+\left|\tilde{\mathbf{v}}_{\mathbf{i}}^{\dagger} \mathbf{h}_{\mathbf{i j}}\right|^{2}\right)^{2}}\left(\mathbf{h}_{\mathbf{i j}} \mathbf{h}_{\mathbf{i j}}^{\dagger}\right)\right]
$$

with eigenvalue $\lambda_{i}$. If $\lambda_{i} \neq 0$, it follows that $\tilde{\mathbf{v}}_{\mathbf{i}}$ must lie in the span of $\left\{\mathbf{h}_{\mathbf{i}}, \mathbf{h}_{\mathbf{i j}}\right\}$. If $\lambda_{i}=0$ for some pair of $\tilde{\mathbf{v}}_{\mathbf{1}}$ and $\tilde{\mathbf{v}}_{\mathbf{2}}$, we can ignore this possibility. The reason is that since the power constraint for user $i$ is inactive, either the conclusion of Proposition 1 (shown below) excludes the possibility that this pair of $\tilde{\mathbf{v}}_{\mathbf{1}}$ and $\tilde{\mathbf{v}}_{\mathbf{2}}$ is optimal, or we can simply assume $\tilde{\mathbf{v}}_{\mathbf{i}}=c \mathbf{h}_{\mathbf{i i}}$ with real coefficient because $\mathbf{h}_{\mathbf{i i}}$ is aligned with $\mathbf{h}_{\mathbf{i j}}$. In another word, the following argument is still valid.

If $\mathbf{h}_{\mathbf{i j}}$ is orthogonal to $\mathbf{h}_{\mathbf{i i}}$, it is easy to see that the optimal beamforming vector $\tilde{\mathbf{v}}_{\mathbf{i}}$ will be aligned with $\mathbf{h}_{\mathbf{i i}}$ and consume all power, i.e., $\tilde{\mathbf{v}}_{\mathbf{i}}=c \mathbf{h}_{\mathbf{i j}}$, where $c$ is some real constant. If not, since $\mathbf{h}_{\mathbf{i j}}$ can be expressed as a linear combination of $\mathbf{h}_{\mathbf{i i}}$ and $P_{\mathbf{h}_{\mathbf{i j}}}^{\perp} \mathbf{h}_{\mathbf{i i}}$, where $P_{\mathbf{x}}^{\perp} \mathbf{y}$ is the orthogonal projection of vector $\mathbf{y}$ onto vector $\mathbf{x}$, and the subspace spanned by $\mathbf{h}_{\mathbf{i i}}$ and $\mathbf{h}_{\mathbf{i j}}$ is equivalent to the subspace spanned by $\mathbf{h}_{\mathrm{ii}}$ and $P_{\mathbf{h}_{\mathbf{i j}}}^{\perp} \mathbf{h}_{\mathrm{ii}}$, then the optimal $\tilde{\mathbf{v}}_{\mathbf{i}}, i=1,2$, can be written as

$$
\begin{aligned}
& \tilde{\mathbf{v}}_{\mathbf{1}}=c_{11} \mathbf{h}_{1 \mathbf{1}}+c_{12} P_{\mathbf{h}_{1 \mathbf{2}}}^{\perp} \mathbf{h}_{\mathbf{1 1}} \\
& \tilde{\mathbf{v}}_{\mathbf{2}}=c_{22} \mathbf{h}_{\mathbf{2 2}}+c_{21} P_{\mathbf{h}_{\mathbf{2 1}}}^{\perp} \mathbf{h}_{\mathbf{2 2}}
\end{aligned}
$$

\footnotetext{
${ }^{3}$ In the remainder of this section, we will follow the convention that index $j$ refers to the interfering user for user $i$ (i.e. $j \neq i$ ).
}

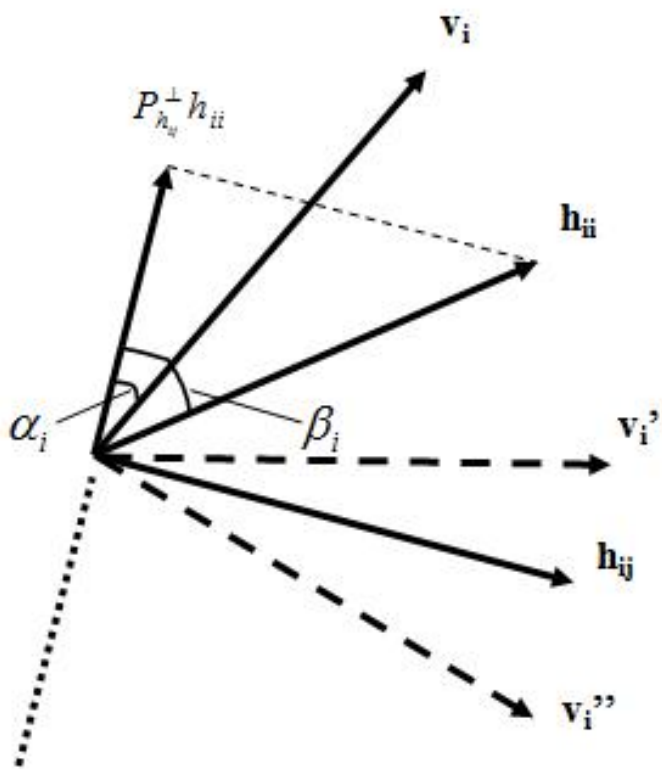

Fig. 1. Illustration of the vectors $\mathbf{h}_{\mathbf{i} \mathbf{i}}, \mathbf{h}_{\mathbf{i j}}, P_{\mathbf{h}_{\mathbf{i j}}}^{\perp} \mathbf{h}_{\mathbf{i}}, \mathbf{v}_{\mathbf{i}}, \mathbf{v}_{\mathbf{i}}^{\prime}$, and $\mathbf{v}_{\mathbf{i}}^{\prime \prime}$ and the angles $\alpha_{i}$ and $\beta_{i}$.

where the $c$ 's are the combining coefficients. Taking $c_{i i}=$ 1 and $c_{i j}=0, i \neq j$, is conventional (channel-matched) beamforming, whereas taking $c_{i i}=0, c_{i j}=1$ is a zeroforcing approach, which cause no interference to the other user. Note that in general neither is globally optimal.

¿From now on, we will focus on a general case, in which $\mathbf{h}_{\mathbf{i j}}$ is not orthogonal to $\mathbf{h}_{\mathbf{i i}}$, i.e., $\mathbf{h}_{\mathbf{i i}} \neq P_{\mathbf{h}_{\mathbf{i j}}}^{\perp} \mathbf{h}_{\mathbf{i i}}$. Later on, it is easy to verify that our results of parametrization can still applied to the orthogonal case.

Without loss of generality, we can assume that $c_{12}$ and $c_{21}$ are real and nonnegative, but that $c_{11}$ and $c_{22}$ are complex (for the time being). Substituting for the optimal $\tilde{\mathbf{v}}_{\mathbf{i}}$ in the objective of the original problem gives

$$
\begin{aligned}
& \text { Total Utility }=u_{1}\left(\frac{\left.\left|c_{11}^{*}\right| \mathbf{h}_{\mathbf{1 1}}\right|^{2}+\left.c_{12}\left(\left|\mathbf{h}_{\mathbf{1 1}}\right|^{2}-\frac{\left|\mathbf{h}_{12}^{\dagger} \mathbf{h}_{11}\right|^{2}}{\left|\mathbf{h}_{\mathbf{1 2}}\right|^{2}}\right)\right|^{2}}{n_{0}+\left|c_{22}\right|^{2}\left|\mathbf{h}_{\mathbf{2 2}}^{\dagger} \mathbf{h}_{\mathbf{2 1}}\right|^{2}}\right) \\
& \quad+u_{2}\left(\frac{\left.\left|c_{22}^{*}\right| \mathbf{h}_{\mathbf{2 2}}\right|^{2}+\left.c_{21}\left(\left|\mathbf{h}_{\mathbf{2 2}}\right|^{2}-\frac{\left|\mathbf{h}_{\mathbf{2}}^{\dagger} \mathbf{h}_{\mathbf{2}}\right|^{2}}{\left|\mathbf{h}_{\mathbf{2 1}}\right|^{2}}\right)\right|^{2}}{n_{0}+\left|c_{11}\right|^{2}\left|\mathbf{h}_{\mathbf{1 1}}^{\dagger} \mathbf{h}_{\mathbf{1 2}}\right|^{2}}\right) \text {. (8) }
\end{aligned}
$$

Proposition 1: For each user $i=1,2$, if $\mathbf{h}_{\mathbf{i i}}$ is not aligned with $\mathbf{h}_{\mathbf{i j}}$, i.e., $\mathbf{h}_{\mathbf{i i}} \neq a \mathbf{h}_{\mathbf{i j}}$, for any real coefficient $a$, then the corresponding power constraint is binding at optimality, i.e., $\left|\tilde{\mathbf{v}}_{\mathbf{i}}\right|^{2}=P_{i}^{\max }$.

The proof follows from the observation that user $i$ can increase its power without increasing interference to user $j$ by adjusting $c_{i j}$. See Appendix A for more details.

Proposition 2: There exists an optimal beamforming vector for which the coefficients $c_{11}, c_{12}, c_{22}$ and $c_{21}$ are all realvalued and nonnegative. In another word, $\tilde{\mathbf{v}}_{\mathbf{i}}$ is in the convex cone spanned by $\mathbf{h}_{\mathbf{i i}}$ and $P_{\mathbf{h}_{\mathbf{i j}}}^{\perp} \mathbf{h}_{\mathbf{i i}}$, shown in Fig. 1 . 
The proof is based on checking the KKT conditions for the optimal beamformer. Namely, we first show that the optimal beamformer for user $i$, which maximizes his signal power given that the interference power to user $j$ is fixed, can be achieved with real $c_{i i}$ and $c_{i j}$. Then we show that these coefficients are nonnegative by excluding the possibility that the optimal $\tilde{\mathbf{v}}_{\mathbf{i}}$ is not in the convex cone spanned by $\mathbf{h}_{\mathbf{i i}}$ and $P_{\mathbf{h}_{\mathrm{ij}}}^{\perp} \mathbf{h}_{\mathrm{ii}}$. See Appendix B for more details.

According to Proposition 2, the original optimization problem is equivalent to finding the optimal nonnegative and real coefficients $c_{11}, c_{12}, c_{22}$ and $c_{21}$ that maximize the total utility. Propositions 1 and 2 imply that we can characterize $\tilde{\mathbf{v}}_{\mathbf{i}}$ with a single angle $\alpha_{i}$. This angle is the same as that in real space, i.e., we view an $N$-dimensional complex vector as a $2 N$-dimensional real vector. If we define the angle between two complex vectors $\mathbf{x}$ and $\mathbf{y}$ as $\alpha$, denoted by $\alpha \equiv \mathbf{x} \wedge \mathbf{y}$, then we have

$$
\cos \alpha=\frac{\operatorname{Re}\left\{\mathbf{x}^{\dagger} \mathbf{y}\right\}}{|\mathbf{x}||\mathbf{y}|} .
$$

Using this definition, we define $\alpha_{i}$ as the angle between the vector $\tilde{\mathbf{v}}_{\mathbf{i}}$ and $P_{\mathbf{h}_{\mathbf{i j}}}^{\perp} \mathbf{h}_{\mathbf{i i}}$. Since the norm of $\tilde{\mathbf{v}}_{\mathbf{i}}$ is fixed, and $\tilde{\mathbf{v}}_{\mathbf{i}}$ is between $\mathbf{h}_{\mathbf{i i}}$ and $P_{\mathbf{h}_{\mathbf{i j}}}^{\perp} \mathbf{h}_{\mathbf{i i}}$, we have $\alpha_{i} \in\left[0, \beta_{i}\right]$, where $\beta_{i}$ is the angle between $\mathbf{h}_{\mathbf{i i}}$ and $P_{\mathbf{h}_{\mathbf{i j}}}^{\perp} \mathbf{h}_{\mathbf{i i}}$. This is illustrated in Fig. 1.

We have

$$
\left\{\begin{aligned}
\alpha_{i} & =\mathbf{v}_{\mathbf{i}} \wedge P_{\mathbf{h}_{\mathbf{i j}}}^{\perp} \mathbf{h}_{\mathbf{i i}} \\
\beta_{i}-\alpha_{i} & =\mathbf{v}_{\mathbf{i}} \wedge \mathbf{h}_{\mathbf{i i}} \\
\beta_{i} & =\mathbf{h}_{\mathbf{i i}} \wedge P_{\mathbf{h}_{\mathbf{i j}}}^{\perp} \mathbf{h}_{\mathbf{i i}}
\end{aligned}\right.
$$

and can therefore write

$$
\begin{aligned}
c_{i i} & =\frac{1}{\sqrt{\left|\mathbf{h}_{\mathbf{i} \mathbf{i}}\right|^{2}-\left|P_{\mathbf{h}_{\mathbf{i j}}}^{\perp} \mathbf{h}_{\mathbf{i i}}\right|^{2}}} \sin \alpha_{i} \\
c_{i j} & =\frac{1}{\left|P_{\mathbf{h}_{\mathbf{i j}}}^{\perp} \mathbf{h}_{\mathbf{i i}}\right|} \cos \alpha_{i}-\frac{1}{\sqrt{\left|\mathbf{h}_{\mathbf{i i}}\right|^{2}-\left|P_{\mathbf{h}_{\mathbf{i j}}}^{\perp} \mathbf{h}_{\mathbf{i}}\right|^{2}}} \sin \alpha_{i} .
\end{aligned}
$$

Substituting for the coefficients $c$ 's in (8) gives ${ }^{4}$

$$
\begin{aligned}
\text { Total Utility }=u_{1} & \left(\frac{\left|\mathbf{h}_{\mathbf{1 1}}\right|^{2} \cos ^{2}\left(\beta_{1}-\alpha_{1}\right)}{n_{0}+\left|\mathbf{h}_{\mathbf{2 1}}\right|^{2} \sin ^{2} \alpha_{2}}\right) \\
& +u_{2}\left(\frac{\left|\mathbf{h}_{\mathbf{2 2}}\right|^{2} \cos ^{2}\left(\beta_{2}-\alpha_{2}\right)}{n_{0}+\left|\mathbf{h}_{\mathbf{1 2}}\right|^{2} \sin ^{2} \alpha_{1}}\right)
\end{aligned}
$$

and the maximization is over $\alpha_{1}$ and $\alpha_{2}$.

\section{B. Parameterized Asynchronous Distributed Algorithm}

With the previous parametrization, we restate the utility maximization problem as

$$
\begin{array}{cc}
\max _{\alpha_{1}, \alpha_{2}} & u_{1}\left(\frac{\left|\mathbf{h}_{\mathbf{1 1}}\right|^{2} \cos ^{2}\left(\beta_{1}-\alpha_{1}\right)}{n_{0}+\left|\mathbf{h}_{\mathbf{2 1}}\right|^{2} \sin ^{2} \alpha_{2}}\right) \\
& +u_{2}\left(\frac{\left|\mathbf{h}_{\mathbf{2 2}}\right|^{2} \cos ^{2}\left(\beta_{2}-\alpha_{2}\right)}{n_{0}+\left|\mathbf{h}_{\mathbf{1 2}}\right|^{2} \sin ^{2} \alpha_{1}}\right) \\
\text { s.t. } & \alpha_{i} \in\left[0, \beta_{i}\right] \quad i=1,2
\end{array}
$$

\footnotetext{
${ }^{4}$ Here, for simplicity, we assume unit power constraints. You can also view it as $P_{i}^{\max }$ is absorbed by channel vector $\mathbf{h}$.
}

where $\beta_{i}=\mathbf{h}_{\mathbf{i i}} \wedge P_{\mathbf{h}_{i j}}^{\perp} \mathbf{h}_{\mathbf{i i}}$. We can now apply the distributed algorithm proposed in Section I by replacing variables $\mathbf{v}_{\mathbf{i}}$ 's with $\alpha_{i}$. The resulting subproblem for each user is

$$
\begin{gathered}
\max _{\alpha_{i}} u_{i}\left(\frac{\left|\mathbf{h}_{\mathbf{i i}}\right|^{2} \cos ^{2}\left(\beta_{i}-\alpha_{i}\right)}{n_{0}+\left|\mathbf{h}_{\mathbf{j i}}\right|^{2} \sin ^{2} \alpha_{j}}\right)-\pi_{j}\left|\mathbf{h}_{\mathbf{i j}}\right|^{2} \sin ^{2} \alpha_{i} \quad\left(P_{a i}\right) \\
\text { s.t. } \quad \alpha_{i} \in\left[0, \beta_{i}\right] .
\end{gathered}
$$

The objective function can be interpreted as a payoff function in a non-cooperative game. That is,

$s_{i}\left(\alpha_{i} ; \alpha_{j}, \pi_{j}\right)=u_{i}\left(\frac{\left|\mathbf{h}_{\mathbf{i i}}\right|^{2} \cos ^{2}\left(\beta_{i}-\alpha_{i}\right)}{n_{0}+\left|\mathbf{h}_{\mathbf{j i}}\right|^{2} \sin ^{2} \alpha_{j}}\right)-\pi_{j}\left|\mathbf{h}_{\mathbf{i j}}\right|^{2} \sin ^{2} \alpha_{i}$,

where $\pi_{j}$ is the interference price announced by user $j$ given by

$$
\pi_{j}=-\frac{\partial u_{j}}{\partial I_{j}}=u_{j}^{\prime} \frac{\left|\mathbf{h}_{\mathbf{j} \mathbf{j}}\right|^{2} \cos ^{2}\left(\beta_{j}-\alpha_{j}\right)}{\left(n_{0}+\left|\mathbf{h}_{\mathbf{i j}}\right|^{2} \sin ^{2} \alpha_{i}\right)^{2}} .
$$

The parameterized asynchronous distributed pricing algorithm is described as follows:

1) Each user $i$ (1 or 2$)$ chooses an initial angle $\alpha_{i}^{0} \in\left[0, \beta_{i}\right]$ and an initial interference price $\pi_{i}^{0} \in$ $\left[0, \max \left\{u_{i}^{\prime}\right\} \frac{\left|\mathbf{h}_{\mathbf{i i}}\right|^{2}}{n_{0}^{2}}\right]$.

2) At each time $n$, one user $i$ is randomly selected to maximize its payoff function $s_{i}\left(\alpha_{i}\right)$ and update its angle $\alpha_{i}$, given the other user's angle $\alpha_{j}$ and prices $\pi_{j}, j \neq i$, i.e.,

$$
\alpha_{i}(n+1)=\underset{\alpha_{i} \in\left[0, \beta_{i}\right]}{\operatorname{argmax}} s_{i}\left(\alpha_{i} ; \alpha_{j}(n), \pi_{j}(n)\right) .
$$

3) Each user $i$ calculates the new interference price $\pi_{i}$ given the current angles $\alpha_{1,2}$ and announces it to the other user.

4) Repeat from step 2.

As in [1], we can view the distributed algorithm as a noncooperative game, denoted by $G$, in which there are four players corresponding to $\left\{\alpha_{1}, \alpha_{2}, \pi_{1}, \pi_{2}\right\}$, and each of them maximizes its own payoff function. The payoff function for $\alpha_{i}$ is given in (13), while the payoff function for $\pi_{i}$ is

$$
s_{\pi_{i}}=-\left(\pi_{i}-u_{i}^{\prime} \frac{\left|\mathbf{h}_{\mathbf{i i}}\right|^{2} \cos ^{2}\left(\beta_{i}-\alpha_{i}\right)}{\left(n_{0}+\left|\mathbf{h}_{\mathbf{j i}}\right|^{2} \sin ^{2} \alpha_{j}\right)^{2}}\right)^{2}
$$

in order that interference price is always updated according to (14).

Proposition 3: The game $G$ can be viewed as a supermodular game with transformed strategies if $-\frac{u_{i}^{\prime \prime} \gamma_{i}}{u_{i}^{\prime}} \in(0,1]$, or $-\frac{u_{i}^{\prime \prime} \gamma_{i}}{u_{i}^{\prime}} \in[1,2]$, for both $i=1,2$, where $\gamma_{i}$ is the received SINR of user $i$.

Proof: In order to show a game is supermodular, we need to verify: a) the strategy space for each player is a nonempty and compact sublattice; b) each payoff function is continuous in all players' strategies; c) each player's payoff function is supermodular in his own strategy; and d) each player's payoff function has increasing differences between any component of his own strategy and any component of any other's strategy. For the game $G$, conditions a) - c) are trivial because each player's strategy space is one-dimensional. 
Condition d) does not hold with the original definition of strategies. However, it can be checked that if $-\frac{u_{i}^{\prime \prime} \gamma_{i}}{u_{i}^{\prime}} \in$ $(0,1]$, then $G$ is supermodular in the transformed strategies $\left(\alpha_{1},-\alpha_{2}, \pi_{1},-\pi_{2}\right)$; if $-\frac{u_{i}^{\prime \prime} \gamma_{i}}{u_{i}^{\prime}} \in[1,2]$, then $G$ is supermodular in the transformed strategies $\left(\alpha_{1}, \alpha_{2},-\pi_{1},-\pi_{2}\right)$.

¿From Theorem 1 in [1], we conclude that the distributed pricing algorithm converges under the following conditions.

Corollary 1: For a two-user MISO network, if both users' utility functions satisfy $-\frac{u_{i}^{\prime \prime} \gamma_{i}}{u_{i}^{\prime}} \in(0,1]$ (or $-\frac{u_{i}^{\prime \prime} \gamma_{i}}{u_{i}^{\prime}} \in[1,2]$ ) and the distributed algorithm is initialized with the smallest (or largest) element in its transformed strategy space, then the strategies $^{5}$ monotonically converge to the component-wise smallest (largest) Nash Equilibrium (NE) which corresponds to a solution to the KKT condition for the original problem.

Specifically, if $-\frac{u_{i}^{\prime \prime} \gamma_{i}}{u_{i}^{\prime}} \in(0,1]$, the smallest element in the strategy space is $\left(\alpha_{1}=0, \alpha_{2}=\beta_{2}, \pi_{1}=0, \pi_{2}=\right.$ $\left.\max \left\{u_{2}^{\prime}\right\} \frac{\left|\mathbf{h}_{\mathbf{2 2}}\right|^{2}}{n_{0}^{2}}\right)$, while the largest element is $\left(\alpha_{1}=\beta_{1}, \alpha_{2}=\right.$ $\left.0, \pi_{1}=\max \left\{u_{1}^{\prime}\right\} \frac{\left|\mathbf{h}_{11}\right|^{2}}{n_{0}^{2}}, \pi_{2}=0\right)$. The initial strategies are similar when $-\frac{u_{i}^{\prime \prime} \gamma_{i}}{u_{i}^{\prime}} \in[1,2]$. Since the theorem in [1] only shows that the strategies are eventually bounded componentwise by the smallest and largest NE, convergence is not guaranteed if each user starts from an arbitrary strategy (selection of beamformers). In other words, without excluding the possibility that there are multiple NE's, to guarantee convergence we must carefully select the initial strategy.

Now, we will show how to solve the subproblem $P_{a i}$ efficiently, i.e., optimize the payoff function for $\alpha_{i}$. Taking the derivative of $s_{i}$ in (13) over $\alpha_{i}$, we obtain

$$
\begin{aligned}
\frac{\partial s_{i}\left(\alpha_{i}\right)}{\partial \alpha_{i}} & =u_{i}^{\prime} \frac{2\left|\mathbf{h}_{\mathbf{i i}}\right|^{2} \cos \left(\beta_{i}-\alpha_{i}\right) \sin \left(\beta_{i}-\alpha_{i}\right)}{n_{0}+\left|\mathbf{h}_{\mathbf{j i}}\right|^{2} \sin ^{2} \alpha_{j}} \\
& -\pi_{j}\left|\mathbf{h}_{\mathbf{i j}}\right|^{2} \sin 2 \alpha_{i} \\
= & 2 u_{i}^{\prime} \gamma_{i} \tan \left(\beta_{i}-\alpha_{i}\right)-\pi_{j}\left|\mathbf{h}_{\mathbf{i j}}\right|^{2} \sin 2 \alpha_{i}
\end{aligned}
$$

where $u_{i}^{\prime}$ and $\gamma_{i}$ (SINR) are both functions of $\alpha_{i}$. It is easy to check that the derivative is positive at $\alpha_{i}=0$ and negative at $\alpha_{i}=\beta_{i}$. Therefore, the optimal solution is not binding. Therefore, we only need to solve the following equation

$$
2 u_{i}^{\prime} \gamma_{i} \tan \left(\beta_{i}-\alpha_{i}\right)=\pi_{j}\left|\mathbf{h}_{\mathbf{i j}}\right|^{2} \sin 2 \alpha_{i}
$$

which is easier than solving subproblem $P_{i}$ with an $N$ dimensional variable, although no closed-form solution is available.

Furthermore, if $-\frac{u_{i}^{\prime \prime} \gamma_{i}}{u_{i}^{\prime}}>1$, i.e., $\frac{\partial u_{i}^{\prime} \gamma_{i}}{\partial \gamma_{i}}=u_{i}^{\prime \prime} \gamma_{i}+u_{i}^{\prime}<0$, then we know $u_{i}^{\prime} \gamma_{i}$ is monotonically decreasing as $\gamma_{i}$ increases, which implies that $u_{i}^{\prime} \gamma_{i}$ decreases as $\alpha_{i}$ increases. Therefore, $2 u_{i}^{\prime} \gamma_{i} \tan \left(\beta_{i}-\alpha_{i}\right)$ is a monotonically decreasing function. Then, it is obvious that the solution to (17) is unique and the payoff function $s_{i}\left(\alpha_{i}\right)$ is quasi-concave. The utility function satisfying $-\frac{u_{i}^{\prime \prime} \gamma_{i}}{u_{i}^{\prime}} \in[1,2]$ fits into this case.

\footnotetext{
${ }^{5}$ Note that, when optimizing the payoff function, if the optimal solution is not unique, both players should pick either the largest (in the transformed strategy space) maximizer or the smallest one uniformly.
}

For a two-user system, the problem can be further simplified if the utility function has the form of $\theta_{i} \log \left(\gamma_{i}\right)$. Then,

$$
\begin{aligned}
\text { Total Utility }=\log & \left(\frac{\left[\left|\mathbf{h}_{\mathbf{1 1}}\right|^{2} \cos ^{2}\left(\beta_{1}-\alpha_{1}\right)\right]^{\theta_{1}}}{\left[n_{0}+\left|\mathbf{h}_{\mathbf{1 2}}\right|^{2} \sin ^{2} \alpha_{1}\right]^{\theta_{2}}}\right) \\
& +\log \left(\frac{\left[\left|\mathbf{h}_{\mathbf{2 2}}\right|^{2} \cos ^{2}\left(\beta_{2}-\alpha_{2}\right)\right]^{\theta_{2}}}{\left[n_{0}+\left|\mathbf{h}_{\mathbf{2 1}}\right|^{2} \sin ^{2} \alpha_{2}\right]^{\theta_{1}}}\right) .
\end{aligned}
$$

After reorganizing, the terms containing $\alpha_{1}$ and $\alpha_{2}$ separate. Therefore each user only needs to optimize the corresponding term regardless of the other's beamformer, which means no iteration is needed. The optimal beamformer for user $i$ in this case is the solution to

$$
2 \theta_{i} \tan \left(\beta_{i}-\alpha_{i}\right)=\theta_{j} \frac{\left|\mathbf{h}_{\mathbf{i j}}\right|^{2} \sin 2 \alpha_{i}}{n_{0}+\left|\mathbf{h}_{\mathbf{i j}}\right|^{2} \sin ^{2} \alpha_{i}} .
$$

The angle parametrization for the two-user MISO system therefore enables a distributed algorithm, which uniquely determines the optimal beamforming vector for each user. Furthermore, the computation in every iteration becomes relatively simple. In Section IV, we compare the original ( $N$-dimensional) and parameterized asynchronous distributed algorithms numerically in terms of convergence rate.

\section{Alternative Game formulation}

In this section we discuss an alternative game formulation of the original MISO-ADP algorithm (discussed in Section II. Using this formulation, we can can again show that for suitable utility functions, the two user algorithm corresponds to best response updates in a supermodular game. Note that the "natural" game formulation for the original MISOADP algorithm does not correspond to a supermodular game because the strategy space for each user's beamformimg vector is not a sublattice. However, in such a game the only aspect of user $i$ 's strategy that effects user $j$ 's strategy choice is the received interference power. Motivated by this, we consider a game where the action chosen by each user is not the beam-forming vector but the interference power at the other user's receiver.

More precisely, let $I_{i j}=\left|\mathbf{v}_{\mathbf{i}}^{\dagger} \mathbf{h}_{\mathbf{i j}}\right|^{2}$ denote the interference power from transmitter $i$ to receiver $j$. Problem $P_{i}$ in Section II is equivalent to the following problem:

$$
\max _{I_{i j} \geq 0} S_{i j}\left(I_{i j}\right)
$$

where

$$
\begin{aligned}
S_{i j}\left(I_{i j}\right):=\max _{\mathbf{v}_{\mathbf{i}}} \quad u_{i}\left(\frac{\left|\mathbf{v}_{\mathbf{i}}^{\dagger} \mathbf{h}_{\mathbf{i i}}\right|^{2}}{n_{0}+I_{j i}}\right)-\pi_{j} I_{i j} \quad\left(P_{I_{i j}}\right) \\
\text { s.t. } \quad\left|\mathbf{v}_{\mathbf{i}}\right|^{2} \leq P_{i}^{\max } \\
\left|\mathbf{v}_{\mathbf{i}}^{\dagger} \mathbf{h}_{\mathbf{i j}}\right|^{2} \leq I_{i j} \quad(j \neq i) .
\end{aligned}
$$

Problem $P_{I_{i j}}$ can be viewed as finding the optimal beamformer satisfying not only the power constraint but also the interference constraint set by $I_{i j}$. Therefore for a given value of $I_{j i}$ an $\pi_{j}$, choosing $I_{i j}$ to maximize $S_{i j}$ is equivalent to selecting a beam $\mathbf{v}_{\mathbf{i}}$ to maximize Problem $P_{i}$. Also note that for any such choice of $I_{i j}$ it must be that the second constraint 
in Problem $P_{I_{i j}}$ is tight and so $I_{i j}$ will in fact be the actual interference power received by user $j$.

Now we can construct a non-cooperative game $G^{\prime}$ with four players corresponding to the variables $I_{i j}, I_{j i}, \pi_{i}$ and $\pi_{j}$. The pay-off of each $I_{i j}$-player is given by $S_{i j}$. The payoff function of each $\pi_{i}$-player is again given by

$$
S_{\pi_{i}}\left(\pi_{i}\right)=-\left(\pi_{i}-u_{i}^{\prime}\left(\frac{\left|\mathbf{v}_{\mathbf{i}}^{\dagger} \mathbf{h}_{\mathbf{i i}}\right|^{2}}{n_{0}+I_{j i}}\right) \frac{\left|\mathbf{v}_{\mathbf{i}}^{\dagger} \mathbf{h}_{\mathbf{i i}}\right|^{2}}{\left(n_{0}+I_{j i}\right)^{2}}\right)^{2},
$$

which is the same as in the game $G$ from the previous section..

Note that now the choice of $\mathbf{v}_{\mathbf{i}}$ now does not correspond to the action of any player in the game, but can still be viewed as an implicit function of corresponding interference power and prices.

Proposition 4: The game $G^{\prime}$ is a supermodular game with the transformed strategies $\left(I_{i j},-I_{j i}, \pi_{i},-\pi_{j}\right)$ if $-\frac{u_{i}^{\prime \prime} \gamma_{i}}{u_{i}^{\prime}} \in$ $(0,1]$, or $\left(I_{i j}, I_{j i},-\pi_{i},-\pi_{j}\right)$ if $-\frac{u_{i}^{\prime \prime} \gamma_{i}}{u_{i}^{\prime}} \in[1,2]$, for both $i=1,2$, where $\gamma_{i}$ is the received SINR of user $i$.

The proof of this again follows from verifying the four conditions listed in the proof of Proposition 3. The details are given in Appendix C.

Based on Proposition 4, as long as the beamformers and interference prices of the original MISO-ADP algorithm are initialized to the smallest (or largest) element in the transformed strategy space ${ }^{6}$ for $G^{\prime}$, then the algorithm will converge. We summarize this in the following corollary.

Corollary 2: For a two-user MISO network, if both users' utility functions satisfy $-\frac{u_{i}^{\prime \prime} \gamma_{i}}{u_{i}^{\prime}} \in(0,1]$, and the original MISO-ADP algorithm is initialized with $\left(\mathbf{v}_{\mathbf{1}}=0, \mathbf{v}_{\mathbf{2}}=\right.$ $\left.c_{2} \mathbf{h}_{\mathbf{2 1}}, \pi_{1}=0, \pi_{2}=\max \left\{u_{2}^{\prime}\right\} \frac{\left|\mathbf{h}_{\mathbf{2 2}}\right|^{2}}{n_{0}^{2}}\right)$ or $\left(\mathbf{v}_{\mathbf{1}}=c_{1} \mathbf{h}_{\mathbf{1 2}}, \mathbf{v}_{\mathbf{2}}=\right.$ $\left.0, \pi_{1}=\max \left\{u_{1}^{\prime}\right\} \frac{\left|\mathbf{h}_{11}\right|^{2}}{n_{0}^{2}}, \pi_{2}=0\right)$, where $c_{1}$ and $c_{2}$ are real coefficients chosen to that the beamformer has maximum power, then the algorithm converges to a Nash Equilibrium that corresponds to a solution to the KKT condition for the original problem. If both users' utility functions satisfy $-\frac{u_{i}^{\prime \prime} \gamma_{i}}{u_{i}^{\prime}} \in[1,2]$, the same results hold result with a different initialization.

\section{Simulation Results}

In this section, we show some typical performance plots for both the generalized asynchronous distributed algorithm in Section II and the parameterized asynchronous distributed algorithm in Section III.

\section{A. Generalized Asynchronous Distributed Algorithm}

In the system there are 5 pairs of transmitters and receivers with 4 transmit antennas each. The users are randomly placed within a square of $1 \mathrm{~km} \times 1 \mathrm{~km}$. Each entry of the channel vector is assumed to be an iid complex Gaussian random variable, where the variance is determined by the distance attenuation. Specifically, the variance of both real

\footnotetext{
${ }^{6}$ If $\mathbf{v}_{\mathbf{i}}=0$, then the corresponding interference power is zero. If $\mathbf{v}_{\mathbf{i}}=$ $c \mathbf{h}_{\mathbf{i j}}$ with appropriate real scalar c to reach the maximum power, then $I_{i j}$ reaches its maximum.
}

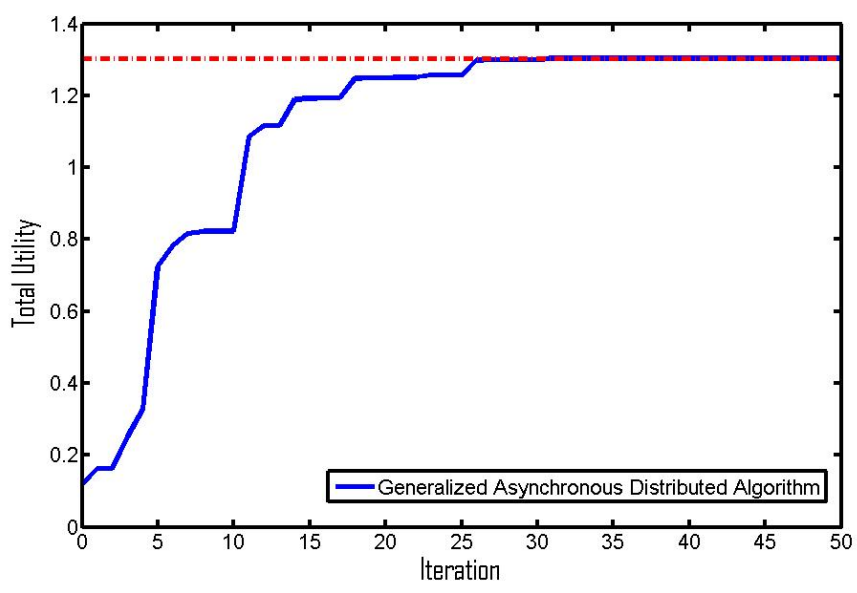

(a) The generalized asynchronous distributed algorithm.

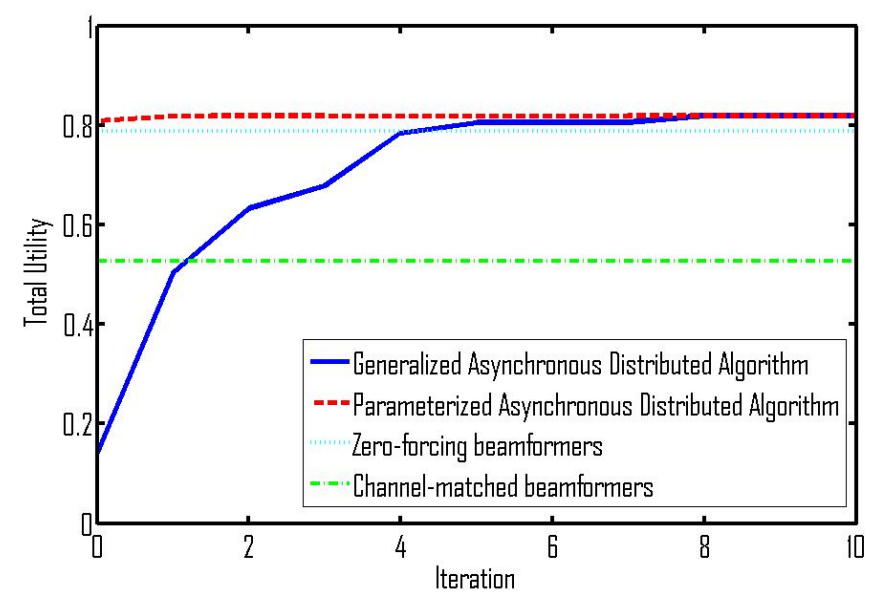

(b) The parameterized asynchronous distributed algorithm.

Fig. 2. Illustration of the performance of two distributed algorithms.

and imaginary parts is $\sigma^{2}(d)=\sigma_{0}^{2}\left(\frac{d}{100}\right)^{-4}$, where $\sigma_{0}^{2}$ is the reference variance at a distance of $100 \mathrm{~m}$, which is the minimum separation between any transmitter and receiver, and $d$ is the separation in meters. We also assume the normalized maximum transmit power for each user is one, $\sigma_{0}^{2}=10^{-7}$, and the variance of noise is $10^{-9}$ so that the average received SNR at $100 \mathrm{~m}$ is 200 (about $23 \mathrm{~dB}$ ). For these results the utility function is $u(\gamma)=\log (1+\gamma)$.

Although in Section II, we are not able to show the convergence of the generalized asynchronous distributed algorithm, it is observed to converge numerically in all cases simulated. Fig. IV-A shows total utility versus number of iterations for a particular model realization. The algorithm starts from a random selection of beamformers, and converges to a stationary point, which is an NE of the corresponding noncooperative game. To check whether the limit point is indeed optimal, we used MATLAB to solve the global optimization problem 
starting from the limit point achieved by the algorithm. The total utility obtained in this way is indicated by the red dashdot line in Fig. IV-A, which matches the limit point. Hence we conclude that the generalized asynchronous distributed algorithm does converge to a local optimum.

\section{B. Parameterized Asynchronous Distributed Algorithm}

Here, we consider a two-user MISO system with the same setting as in the previous subsection. Applying the parameterized asynchronous distributed algorithm proposed in Section III, we optimize the total utility iteratively. The numerical results show that the algorithm still converges even if we start from any arbitrary angle satisfying the constraints. As in Fig. IV-A, Fig. IV-A also shows total utility versus number of iterations for a particular model realization, which illustrates the convergence of the algorithm. Furthermore, we compare it with the generalized asynchronous distributed algorithm. Both algorithms start from random beamformers or angles ${ }^{7}$. It is easy to see that the parameterized asynchronous distributed algorithm yields a higher initial total utility, and it converges more rapidly. The main reason is because we narrow the domain of potential beamformers by angle parametrization. The preceding observation is quite typical after trying many model realizations. When averaged over 100 model realizations, the results show that it takes 4.28 iterations for the parameterized asynchronous distributed algorithm to converge ${ }^{8}$, while for the generalized asynchronous distributed algorithm, it takes 6.0 iterations for convergence. In Fig. IV-A, we also show the total utility with the (suboptimal) zero-forcing and channel-matched filters.

\section{Conclusions}

We have presented two distributed algorithms for selecting each user's beamforming vector to maximize the total utility. The generalized asynchronous distributed algorithm is an extension of the asynchronous distributed pricing algorithm in [1]. (See also [3].) In that algorithm, each user announces an interference price, which represents his current marginal cost per unit interference power. After exchanging interference prices, users update their beamforming vectors by maximizing a local payoff function. Although we have not proved that this algorithm converges with more than two users, numerical results indicate that it performs quite well. For a two-user system we have presented two proofs that this algorithm converges: one based on an angle parameterization and one based on viewing interference power as the strategic variable.

Unfortunately, both the angle parametrization we apply in Section III and the proof using interference power appear to be difficult to generalize to multi-user systems. An open issue, then, is how to prove the convergence of the generalized asynchronous distributed algorithm for multi-user

\footnotetext{
${ }^{7}$ Since an arbitrary beamforming vector $\mathbf{v}_{\mathbf{i}}$ may not lie in the convex cone spanned by $\mathbf{h}_{\mathbf{i i}}$ and $\mathbf{h}_{\mathbf{i i}}$, we cannot ensure that the two algorithms start with the same beamformers.

${ }^{8}$ Numerically, we define convergence as the minimum number of iterations for which the difference in total utility between two consecutive iterations is no greater than some threshold ( $0.1 \%$ in our simulation).
}

systems. Furthermore, it still unknown if the optimum is unique. Extensions to MIMO channels are also interesting for future work.

\section{APPENDIX A}

\section{PROOF OF PROPOSITION 1}

Proof: Suppose that the optimal $\left|\tilde{\mathbf{v}}_{\mathbf{i}}\right|^{2}<P_{i}^{\max }$ for some $i$. Then fixing the corresponding coefficient $c_{i i}$, which means the interference power to the other user will be fixed, we can always increase user $i$ 's signal power by adjusting $c_{i j}$. That is, if $\operatorname{Re}\left\{c_{i i}^{*}\left|\mathbf{h}_{\mathbf{1 1}}\right|^{2}+c_{i j}\left(\left|\mathbf{h}_{\mathbf{i i}}\right|^{2}-\frac{\left|\mathbf{h}_{\mathbf{i j}}^{\dagger} \mathbf{h}_{\mathbf{i i}}\right|^{\mathbf{2}}}{\left|\mathbf{h}_{\mathbf{i j}}\right|^{2}}\right)\right\} \geq 0$, increase $c_{i j}$ within the power constraint; otherwise, decrease $c_{i j}$. Note that the assumption that $\mathbf{h}_{\mathbf{i i}}$ is not aligned with $\mathbf{h}_{\mathbf{i j}}$ implies $\left|\mathbf{h}_{\mathbf{i i}}\right|^{2}-\frac{\left|\mathbf{h}_{\mathbf{i j}}^{\dagger} \mathbf{h}_{\mathbf{i i}}\right|^{2}}{\left|\mathbf{h}_{\mathbf{i j}}\right|^{2}}>0$, i.e., $c_{i j}$ has a non-trivial form.

\section{APPENDIX B}

\section{PROOF OF PROPOSITION 2}

Proof: Consider the optimal beamforming vector $\tilde{\mathbf{v}}_{\mathbf{i}}$ for user $i$. The result is trivial if $\mathbf{h}_{\mathbf{i i}}$ is aligned with $\mathbf{h}_{\mathbf{i j}}$ or it is the case that either $c_{i i}=0$ or $c_{i j}=0$ at optimality. Then suppose that $\left|c_{i i}\right|>0$ and $c_{i j}>0$, and write $c_{i i}=c_{i i}^{\prime} e^{j \theta_{i i}}$, where $c_{i i}^{\prime}$ is real and positive.

If we fix $c_{i i}^{\prime}$ and optimize over $\theta_{i i}$ and $c_{i j}$, then we claim that the optimal $\theta_{i i}$ is 0 or $\pi$. This is because when $c_{i i}^{\prime}$ is fixed, the interference power to user $j$ is $\left|c_{i i}\right|^{2}\left|\mathbf{h}_{\mathbf{i i}}^{\dagger} \mathbf{h}_{\mathbf{i j}}\right|^{2}$, which is fixed, and $\theta_{i i}$ and $c_{i j}$ can only influence the signal power of user $i$. Therefore this problem reduces to:

$$
\begin{gathered}
\max _{c_{i j}, \theta_{i i}}\left|\mathbf{v}_{\mathbf{i}}^{\dagger} \mathbf{h}_{\mathbf{i i}}\right|^{2} \\
\text { s.t. } \quad\left|\mathbf{v}_{\mathbf{i}}\right|^{2}=P_{i}^{\max }
\end{gathered}
$$

which can be further simplified as

$$
\begin{array}{r}
\max _{c_{i j}, \theta_{i i}} c_{i j}^{2} \frac{\left|P_{\mathbf{h}_{\mathbf{i j}}}^{\perp} \mathbf{h}_{\mathbf{i i}}\right|^{2}}{\left|\mathbf{h}_{\mathbf{i i}}\right|^{2}}+2 c_{i i}^{\prime} c_{i j} \cos \theta_{i i} \\
\text { s.t. } \quad c_{i j}^{2}+2 c_{i i}^{\prime} c_{i j} \cos \theta_{i i}=\text { constant. }
\end{array}
$$

The KKT conditions are

$$
\left\{\begin{array}{l}
2 c_{i j} \frac{\left|P_{\mathbf{h}_{\mathbf{i j}}}^{\perp} \mathbf{h}_{\mathbf{i i}}\right|^{2}}{\left|\mathbf{h}_{\mathbf{i i}}\right|^{2}}+2 c_{i i}^{\prime} \cos \theta_{i i}+\lambda_{i}\left(2 c_{i j}+2 c_{i i}^{\prime} \cos \theta_{i i}\right)=0 \\
-2 c_{i i}^{\prime} c_{i j}\left(1+\lambda_{i}\right) \sin \theta_{i i}=0 \\
c_{i j}^{2}+2 c_{i i}^{\prime} c_{i j} \cos \theta_{i i}=\text { constant }
\end{array}\right.
$$

where $\lambda_{i}$ is the Lagrange multiplier, and the unique solution is $\sin \theta_{i i}=0$, i.e, $\theta_{i i}=0$ or $\pi$. Therefore $c_{i i}$ is real.

Now, we can assume all these coefficients are real. Then we want to exclude the possibility that the optimal $\tilde{\mathbf{v}}_{\mathbf{i}}$ is not in the convex cone spanned by $\mathbf{h}_{\mathbf{i i}}$ and $P_{\mathbf{h}_{\mathbf{i j}}}^{\perp} \mathbf{h}_{\mathbf{i i}}$. Without loss of generality, we can assume $c_{i i}>0$ and only consider the right half-plane in Fig. 1. Since $\left|\mathbf{v}_{\mathbf{i}}\right|^{2}=P_{i}^{\max }$, we know the angle between $\mathbf{v}_{\mathbf{i}}$ and $\mathbf{h}_{\mathbf{i i}}$ will uniquely decide its own signal power, while the angle between $\mathbf{v}_{\mathbf{i}}$ and $\mathbf{h}_{\mathbf{i j}}$ will decide the interference power to user $j$. It can be shown that if $\mathbf{v}_{\mathbf{i}}$ is out of the cone (e.g., $\mathbf{v}_{\mathbf{i}}^{\prime}$ or $\mathbf{v}_{\mathbf{i}}^{\prime \prime}$ in Fig. 1) it cannot be a candidate of the optimal $\tilde{\mathbf{v}}_{\mathbf{i}}$ because we can always find another $\mathbf{v}_{\mathbf{i}}$ which will yield either a higher signal power or a lower interference power. 


\section{APPENDIX C \\ PROOF OF PROPOSITION 4}

Proof: For this proof we again verify conditions a)d) given in the proof of Proposition 3. Conditions a) - c) are trivial because each player's strategy space is still onedimensional. For condition d), we need to make sure increasing differences are satisfied between any pair of transformed strategies. If $-\frac{u_{i}^{\prime \prime} \gamma_{i}}{u_{i}^{\prime}} \in(0,1]$, i.e., $u_{i}^{\prime \prime} \gamma_{i}+u_{i}^{\prime} \geq 0$, in order to show increasing differences hold with the transformed strategies $\left(I_{i j},-I_{j i}, \pi_{i},-\pi_{j}\right)$, we need to check the following four inequalities for any strategy choices $\underline{I}_{i j}<\bar{I}_{i j}, \underline{I}_{j i}<\bar{I}_{j i}$, and $\underline{\pi}_{i}<\bar{\pi}_{i}$ :

(a) $S_{i j}\left(\bar{I}_{i j} ; \bar{\pi}_{j}\right)-S_{i j}\left(\bar{I}_{i j} ; \underline{\pi}_{j}\right) \leq S_{i j}\left(\underline{I}_{i j} ; \bar{\pi}_{j}\right)-S_{i j}\left(\underline{I}_{i j} ; \underline{\pi}_{j}\right)$

(b) $S_{i j}\left(\bar{I}_{i j} ; \bar{I}_{j i}\right)-S_{i j}\left(\bar{I}_{i j} ; \underline{I}_{j i}\right) \leq S_{i j}\left(\underline{I}_{i j} ; \bar{I}_{j i}\right)-S_{i j}\left(\underline{I}_{i j} ; \underline{I}_{j i}\right)$

(c) $S_{\pi_{i}}\left(\bar{\pi}_{i} ; \bar{I}_{i j}\right)-S_{\pi_{i}}\left(\bar{\pi}_{i} ; \underline{I}_{i j}\right) \geq S_{\pi_{i}}\left(\underline{\pi}_{i} ; \bar{I}_{i j}\right)-S_{\pi_{i}}\left(\underline{\pi}_{i} ; \underline{I}_{i j}\right)$

(d) $S_{\pi_{i}}\left(\bar{\pi}_{i} ; \bar{I}_{j i}\right)-S_{\pi_{i}}\left(\bar{\pi}_{i} ; \underline{I}_{j i}\right) \leq S_{\pi_{i}}\left(\underline{\pi}_{i} ; \bar{I}_{j i}\right)-S_{\pi_{i}}\left(\underline{\pi}_{i} ; \underline{I}_{j i}\right)$

Inequality (a) is equivalent to

$$
-\bar{I}_{i j}\left(\bar{\pi}_{j}-\underline{\pi}_{j}\right) \leq-\underline{I}_{i j}\left(\bar{\pi}_{j}-\underline{\pi}_{j}\right)
$$

which is obviously true.

Let $\bar{s}$ denote the optimal signal power of user $i$ by solving Problem $P_{I_{i j}}$ given $\bar{I}_{i j}$, and $\underline{s}$ denote the optimal signal power given $\underline{I}_{i j}$. Then inequality (b) can be expressed as

$$
\begin{aligned}
& u_{i}\left(\frac{\bar{s}}{n_{0}+\bar{I}_{j i}}\right)-u_{i}\left(\frac{\bar{s}}{n_{0}+\underline{I}_{j i}}\right) \\
& \quad \leq u_{i}\left(\frac{\underline{s}}{n_{0}+\bar{I}_{j i}}\right)-u_{i}\left(\frac{\underline{s}}{n_{0}+\underline{I}_{j i}}\right),
\end{aligned}
$$

which can be further written as

$$
\begin{aligned}
\int_{\underline{I}_{j i}}^{\bar{I}_{j i}} & -u_{i}^{\prime}\left(\frac{\bar{s}}{n_{0}+I_{j i}}\right) \frac{\bar{s}}{\left(n_{0}+I_{j i}\right)^{2}} \mathrm{~d} I_{j i} \\
& \leq \int_{\underline{I}_{j i}}^{\bar{I}_{j i}}-u_{i}^{\prime}\left(\frac{\underline{s}}{n_{0}+I_{j i}}\right) \frac{\underline{s}}{\left(n_{0}+I_{j i}\right)^{2}} \mathrm{~d} I_{j i} .
\end{aligned}
$$

Using the assumption that $u_{i}^{\prime \prime} \gamma_{i}+u_{i}^{\prime} \geq 0$ implies that

$$
\begin{aligned}
& -u_{i}^{\prime}\left(\frac{\bar{s}}{n_{0}+I_{j i}}\right) \frac{\bar{s}}{\left(n_{0}+I_{j i}\right)^{2}} \\
& \quad \leq-u_{i}^{\prime}\left(\frac{\underline{s}}{n_{0}+I_{j i}}\right) \frac{\underline{s}}{\left(n_{0}+I_{j i}\right)^{2}}
\end{aligned}
$$

Therefore, (20) holds and hence so does inequality (b).

Similar to inequality (b), we can show inequality (c) holds by using (21).

Since the optimal $\mathbf{v}_{\mathbf{i}}$ only depends on $I_{i j}$, then $S_{\pi_{i}}$ can be viewed as a continuous function with respect to $\pi_{i}$ and $I_{j i}$. Furthermore, using that $\frac{\partial S_{\pi_{i}}}{\partial \pi_{i} \partial I_{j i}} \leq 0$, we know inequality (d) holds.

Similar arguments apply for the case where $-\frac{u_{i}^{\prime \prime} \gamma_{i}}{u_{i}^{\prime}} \in[1,2]$.

\section{REFERENCES}

[1] J. Huang, R. A. Berry, and M. L. Honig, "Distributed interference compensation for wireless networks," IEEE Journal on Selected Areas in Communications, vol.24, no. 5, pp.1074-1084, May 2006

[2] C. Shi, R. A. Berry, and M. L. Honig, "Distributed Interference Pricing for OFDM Wireless Networks with Non-Separable Utilities," in Proc. Conference on Information Sciences and Systems, March 2008.

[3] D. A. Schmidt, A. Grundinger, W. Utschick, and M. L. Honig, "Distributed precoder optimization for interfering MISO channels," in International ITG Workshop on Smart Antennas, Feb. 2008, pp. 285290.

[4] M. Chiang, "Balancing Transport and Physical Layers in Wireless Multihop Networks: Jointly Optimal Congestion Control and Power Control," IEEE J. Sel. Areas Comm, vol. 23, no. 1, pp. 104-116, Jan. 2005.

[5] J. Yuan and W. Yu, "Distributed cross-layer optimization of wireless sensor networks: A game theoretic approach," in Proc. IEEE Conf. Global Commun., Nov. 2006.

[6] M. F. Demirkol and M. A. Ingram, "Power-controlled capacity for interfering MIMO links," in Proc. IEEE Vehicular Technology Conference, vol. 1, Oct. 2001

[7] S. Ye and R. S. Blum, "Optimized signalling for MIMO interference systems with feedback," IEEE Trans. Signal Processing, vol. 51, no. $11,2003$.

[8] G. Scutari, D. P. Palomar and S. Barbarossa, "Competitive Design of Multiuser MIMO Systems based on Game Theory: A Unified View," to appear in IEEE Journal on Selected Areas in Communications: Special Issue on Game Theory, Sept. 2008.

[9] E. A. Jorswieck and E. G. Larsson, "The MISO interference channel from a game-theoretic perspective: A combination of selfishness and altruism achieves pareto optimality," in Proc. ICASSP, 2008, pp. 53645367.

[10] D. Bertsekas, Nonlinear Programming, 2nd ed. Belmont, MA: Athena Scientific, 1999 